\title{
Modeling Shrimp Biomass and Viral Infection for Production of Biological Countermeasures
}

\author{
H. T. Banks ${ }^{\mathrm{a}}$, V. A. Bokil ${ }^{\mathrm{b}}$, S. Huc ${ }^{\mathrm{c}}$, A. K. Dhar ${ }^{\mathrm{d}}$, \\ R. A. Bullis ${ }^{\mathrm{e}}$, C. L. Browdy ${ }^{\mathrm{f}}$ and F.C.T. Allnutt ${ }^{\mathrm{g}}$ \\ Center for Research in Scientific Computation ${ }^{\mathrm{a}, \mathrm{b}, \mathrm{c}}$ \\ North Carolina State University \\ Box 8205 \\ Raleigh, NC 27695-8205 \\ Advanced Bionutrition Corporation ${ }^{\mathrm{d}, \mathrm{e}, \mathrm{g}}$ \\ 6430 Dobbin Road \\ Columbia, MD 21045 \\ Marine Resources Research Institute ${ }^{\mathrm{f}}$ \\ South Carolina Department of Natural Resources \\ 217 Ft. Johnson Rd. (P.O. Box 12559) \\ Charleston, SC 29422
}

December 9, 2005

\begin{abstract}
In this paper we develop a mathematical model for the rapid production of large quantities of therapeutic and/or preventative countermeasures. We couple equations for biomass production with those for vaccine production in shrimp that have been infected with a recombinant viral vector expressing a foreign antigen. The model system entails both size and class-age structure.

Key Words: Size/class-age structured population models, shrimp growth/viral progression dynamics, latently-acutely infected.

ahtbanks@ncsu.edu, bvabokil@ncsu.edu, ${ }^{\mathrm{c}}$ shu3@ncsu.edu, dadhar@abn-corp.com,

erbullis@abn-corp.com, ${ }^{\mathrm{f}}$ browdyc@dnr.sc.gov, ${ }^{\mathrm{g}}$ tallnutt@abn-corp.com
\end{abstract}




\section{Introduction}

We consider a novel approach for developing a stable operational platform for the rapid production of large quantities of therapeutic and/or preventative countermeasures. The ideas developed here can also serve as the foundations in designing an economical platform for the production of complex protein therapeutics to replace mammalian cell culture production methods used in the pharmaceutical industry. This approach involves recruiting the biochemical machinery in an existing biomass for the production of a vaccine or antibody by infection using a virus carrying a passenger gene for the desired countermeasure. While our motivation derives from efforts related to first response to deliberate bio toxic attacks on populations, the models we develop may also have use in designing prophylactic production systems against epidemics originating naturally in populations which, without intervention, might result in pandemics. While our model is specific to virus growth and vaccine production in shrimp, the implications for other crustaceans are obvious. And of course the shrimp models we investigate can serve as a foundation for understanding viral progression in other species important to marine agriculture.

Our goal is to model a system wherein one uses shrimp as a scaffold organism to produce biological countermeasures. In such a system one might first stock shrimp postlarvae and allow them to grow normally in a controlled environment. Then one infects them with a recombinant viral vector (e.g., recombinant Taura Syndrome virus or rTSV) expressing a foreign antigen, resulting in vaccine production in live infected shrimp.

To mathematically demonstrate the feasibility of this approach we consider a hybrid model of the shrimp biomass/countermeasure production system which has two components: biomass production, and production of countermeasure (antibody/vaccine). We feed the output of the biomass production model as input to the vaccine production model. For initial investigations the amount of vaccine produced is assumed equal to the total infected biomass. Thus, the vaccine production model will essentially follow the course of the viral dynamics in shrimp.

There is considerable literature on shrimp growth dynamics (e.g., [10, 11, 17, 24]) where mathematical descriptions of growth curves are fitted to data obtained for a specific subspecies of shrimp and no structural information is utilized. However, there is little information in the literature on modeling the dynamics of shrimp at the population level. In [9], the authors incorporate abiotic factors such as temperature into a logistic type growth equation to model biomass dynamics in shrimp; in such models it is assumed that all individuals are identical in characteristics and behavior. However, disregarding structure in constructing mathematical models for the dynamics of shrimp is unrealistic, since shrimp have size dependent characteristics and responses to external environment. The model in this paper is based on the classical McKendrickvon-Foerster/Sinko-Streifer size-structured population equations [18, 21, 23] with mass as the structure variable, i.e., we equate the size variable with the mass in our model.

There also appears to be a dearth of literature on modeling epidemics in shrimp populations. In [20] the authors develop a non-structured five compartment epidemic model of TSV that includes a Reed-Frost transmission process in closed populations of shrimp (Litopenaeus vannamei). However, as in the case of the biomass model, structure can play an important role in the study of viral epidemiology in shrimp. Moreover, experimental results [14] suggest that the mortality rate in acutely infected 
shrimp depends on the length of time that the shrimp remain acute. Also, individuals in the latent phase have varying residency times before they progress into the acute phase. To incorporate all of these features, we attempted to model the progression of TSV in shrimp in a system of delay PDEs. However, it is difficult to correctly account for the different residency periods of individual shrimp in this fashion as the size of the shrimp is a function of time. Instead of tracing back in time to incorporate delays, a different approach involves recording the variable residency times in the different stages by introducing a new variable which we call the class age of an individual. The class age of an individual in a given stage represents the length of time that the individual spends in that stage. Ours is the first attempt at a mathematical model that incorporates size and class age to study the progression of TSV infection in shrimp. A similar approach has been used previously to investigate a linear cell population model. In such models, cells are assumed capable of simultaneous proliferation and maturation where in the proliferating phase, cells are committed to undergo cell division some time units after entering this phase [1]. There are several papers that investigate structured population models with multiple internal variables, for example, see [15, 22].

The outline of the paper is as follows: In Section 2 we plot the growth data observed in several different shrimp populations cultured in super-intensively stocked raceways production systems at the Waddell Mariculture Center in Bluffton, SC. We use regression analysis to fit linear, cubic and exponential growth rate models to this data. In Section 3 we formulate a biomass production model for the normal growth of shrimp and present results of numerical simulations of this model, while in Section 4 we construct a three compartment vaccine production model and analyze this model using the method of characteristics. We also construct a discrete scheme for the vaccine production model. Numerical simulations for the coupled biomass-vaccine production model are discussed in Section 5.

Successful implementation of this shrimp-based expression system could potentially provide an inexpensive alternative method to mammalian cell culture for making complex recombinant proteins. In this context, complex recombinant proteins are ones that undergo post-translational modifications such as glycosylation that cannot easily be duplicated in bacterial and yeast protein expression systems. The shrimp expression system could dramatically lower the costs of production of these complex proteins.

\section{Data Fitting}

We have fitted linear, cubic and exponential solutions $x$ for individual growth rate models $\frac{d x}{d t}=g(x, t)$ (corresponding to $g$ constant, quadratic and exponential) to the data for the mean weight of the shrimp measured during a series of grow out runs in a super-intensively stocked demonstration commercial scale raceway production system [7] at the Waddell Mariculture Center. Growth data is based on population sampling from a series of demonstration runs in a new advanced greenhouse enclosed biosecure system developed for the culture of marine shrimp Litopenaeus vannamei. The Waddell system provides for the culture of shrimp at very high densities without water exchange. A dense microbial floc develops in the water column providing for the recycling of waste material within the system to maintain water quality and enhance shrimp growth. By applying filtration, oxygen injection and well designed feeds and feeding regimes, 
genetically selected specific pathogen free strains of shrimp can be cultured in this system at high densities with good survival and excellent growth.

The various raceway runs initially had different stocking densities. The mean weight $x$ (in gm) of the shrimp were recorded on different days over several weeks. The results of the regression analysis are presented below.

In raceway runs 6 and 9 , the mean stocking weight is $1 \mathrm{gm}$, and linear fits give fair approximations to the data obtained from these runs as observed in Figures 1 and 3, respectively. We can also see in these two figures that a cubic fit gives a much better approximation to the data than the corresponding linear fits. Run 10 was stocked with shrimp that had a mean weight of $4 \mathrm{gm}$, and a linear fit seems to be appropriate in this case as seen in Figure 4 (left). Run 8 was stocked with shrimp that had a mean weight of $0.01 \mathrm{gm}$. From Figure 2 we can see that a linear fit does not do justice to the data. However, a cubic fit works well in this case. In all these figures, the horizontal axis represents time $t$ in days and the vertical axis represents the mean weight $x$ of the shrimp. Each figure also records the equation for the corresponding linear, cubic or exponential fit as the case may be.

We can conclude that a linear fit is a good approximation to the data in the range 1-20 gm, but a linear fit does not approximate the data in the interval 0-20 gm. In [12], the authors have observed that growth of early postlarvae was exponential rather than linear. However, we were unable to use an exponential function to fit the entire data, i.e., in the range 0-20 gm. For example, Figure 4 (right) depicts the best exponential fit to the data obtained from run 8. We can see that this fit does not give a good approximation to the data. Instead a cubic fit seems to yield a good approximation as seen in Figure 2 (right).

Using a linear fit for the mean weight $x$ of the shrimp as a function of time $t$ implies that the growth function $g(x, t)$ is a constant in the interval 1-20 gm. This is the approximation that we have used to perform our simulations. Since a cubic fit (for $x$ as a function of time $t$ ) gives a far better approximation to the data, as seen in Figures 1,2 , and 3 , the growth function $g(x, t)$ would then be a quadratic function of time $t$. We have as yet not used such an approximation in our simulations since we have assumed a mean stocking weight of $1 \mathrm{gm}$.
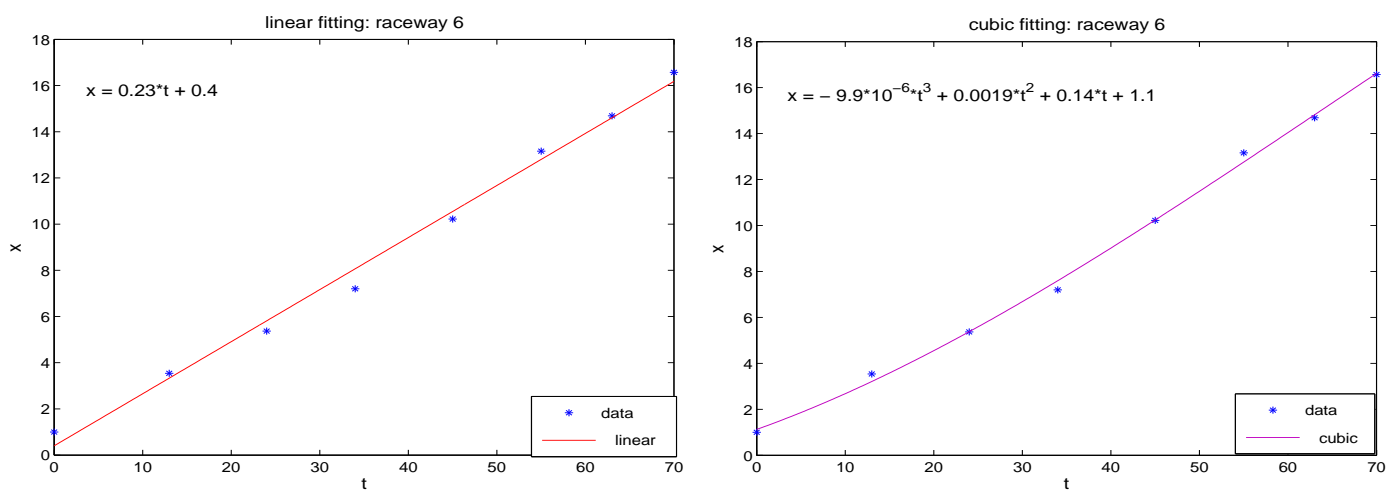

Figure 1: Linear and cubic fitting to the data of raceway run 6 , where the mean stocking weight of the shrimp is $1 \mathrm{gm}$. 

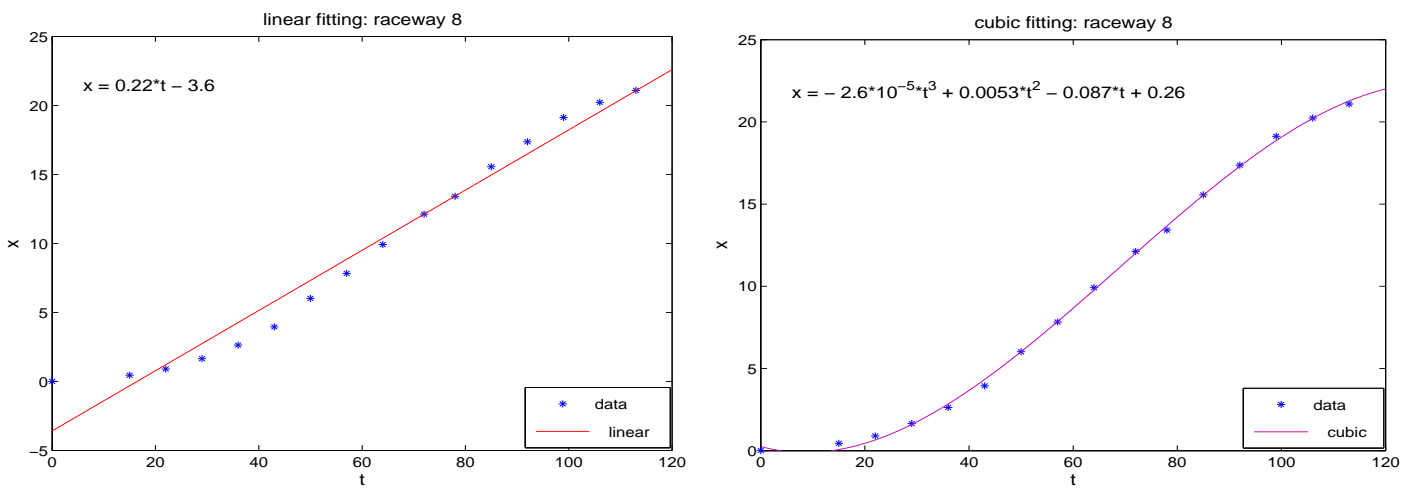

Figure 2: Linear and cubic fitting to the data of raceway run 8, where the mean stocking weight of the shrimp is $0.01 \mathrm{gm}$.
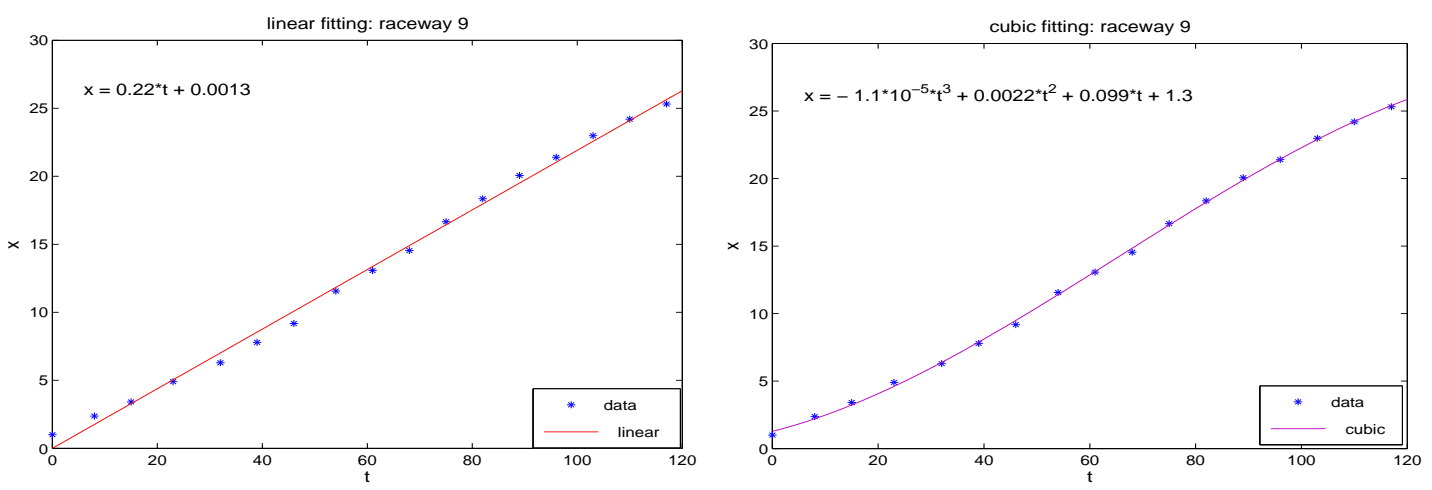

Figure 3: Linear and cubic fitting to the data of raceway run 9, where the mean stocking weight of the shrimp is $1 \mathrm{gm}$.


Figure 4: (left) Linear fitting to the data of raceway run 10, where the mean stocking weight of the shrimp is $4 \mathrm{gm}$. (right) Exponential fitting to the data of raceway run 8, where the mean stocking weight of the shrimp is $0.01 \mathrm{gm}$.

\section{Biomass Production Model}

We present a classical size-structured population model with mass as the structure variable. The factors considered here that affect the total biomass are the growth and 
mortality rates of normal shrimp. Since we have a controlled environment for growth, we may assume that the growth and mortality rates of normal shrimp are not affected by changes in environmental factors such as temperature, as well as other factors such as population density.

Growth in shrimp consists of periods involving molting separated by intermolt periods where no external growth occurs (see [8] for more details). This makes the growth in shrimp a discontinuous process. However, viewed over a long period of time we can approximate the growth as a continuous process. Hence, we assume the individual growth rate $g(x, t)$ to be a continuous function of mass $x$ and time $t$ as depicted in Section 2. The time interval for normal growth that we consider here is not sufficiently long for the shrimp postlarve to grow to adult shrimp and thus we do not consider reproduction in the biomass and vaccine production models.

Based on the above discussion our biomass production model is given by

$$
\begin{aligned}
& u_{t}+(g(x, t) u)_{x}+m(x, t) u=0, \quad(x, t) \in\left(0, x_{\max }\right] \times\left(0, T_{B}\right], \\
& u(0, t)=0, \quad t \in\left(0, T_{B}\right], \\
& u(x, 0)=u^{0}(x), \quad x \in\left[0, x_{\max }\right] .
\end{aligned}
$$

Here $u(x, t)$ denotes density of individuals (number/unit mass) having mass $x$ in gms at time $t$. The growth rate is denoted by $g(x, t)$ (mass/unit time), and the function $m(x, t)$ denotes the mortality rate (1/unit time). The initial population density is given by $u^{0}(x)$ and $x_{\max }$ is the maximum mass of shrimp in the time interval from 0 to a final time $T_{B}$.

There are numerous papers that consider wellposedness of solutions to models similar to (3.1). For example, when the growth rate $g$ and the mortality rate $m$ are functions of the size $x$ only, wellposedness results are proved in [3] using weak formulations, and in [4] semigroup theory is used to prove existence and uniqueness of solutions. For systems with time dependent coefficients an analogous approach using the theory of evolution operators $[6,19]$ offers a means for establishing the desired wellposedness results.

\subsection{Numerical Scheme}

The following notation will be used throughout this section: $\triangle x=x_{\max } / n_{x}$ and $\triangle t=$ $T_{B} / n_{t}$ denote the spatial and time mesh size, respectively. The mesh points are given by: $x_{j}=j \triangle x, j=0,1, \cdots, n_{x}$ and $t_{k}=k \Delta t, k=0,1, \cdots, n_{t}$. We denote by $u_{j}^{k}$,

$g_{j}^{k}$ and $m_{j}^{k}$ the finite difference approximations of $u\left(x_{j}, t_{k}\right), g\left(x_{j}, t_{k}\right)$ and $m\left(x_{j}, t_{k}\right)$, respectively. We adopt the following implicit difference scheme to solve the model (3.1):

$$
\begin{aligned}
& \frac{u_{j}^{k+1}-u_{j}^{k}}{\Delta t}+\frac{g_{j}^{k+1} u_{j}^{k+1}-g_{j-1}^{k+1} u_{j-1}^{k+1}}{\Delta x}+m_{j}^{k+1} u_{j}^{k+1}=0,1 \leq j \leq n_{x} ; 0 \leq k \leq n_{t}-1, \\
& u_{0}^{k+1}=0 .
\end{aligned}
$$

From the above scheme we note that the solution remains positive for all time. This is important from the biological point of view. 


\subsection{Numerical Simulations}

For numerical simulations with (3.2), we assume that $x_{\max }=10 \mathrm{gms}, T_{B}=21$ days, i.e., 3 weeks. The mesh size for $\Delta x$ and $\Delta t$ are taken to be 0.02 and 0.01 , respectively. The initial function $u^{0}(x)$ is defined as $u^{0}(x)=167650 \delta_{1}(x)$. This implies that the initial population only contains shrimp that are $1 \mathrm{gm}$ in mass, and the total number of shrimp that are used to stock the raceway are

$$
\int_{0}^{10} u^{0}(x) d x=167650 \text { shrimp }
$$

which are $1 \mathrm{gm}$ in mass. Hence, the total biomass is $167650 \mathrm{gm}$. Note that the stocking weight of the shrimp is $1 \mathrm{gm}$. Thus, considering the data fits of Section 2, we can assume that the growth rate is a constant function, and it is chosen as $g(x, t)=0.214$. The mortality rate function is chosen as $m(x, t)=0.0014$. Graphs of typical solutions are given in Figures 5 and 6 . In Figure 5 we plot the population density $u$ at time $T_{B}$ as a function of the size $x$. From this figure we see that at time $t=T_{B}$ the weight of most shrimp is around $5.5 \mathrm{gm}$. In Figure 6 we plot the population density $u$ as a function of time $t$ and size $x$, where a cross section $u(x, t)$ for a given time $t$ has a similar shape as $u\left(x, T_{B}\right)$ in Figure 5 centered at a different value of the size $x$.

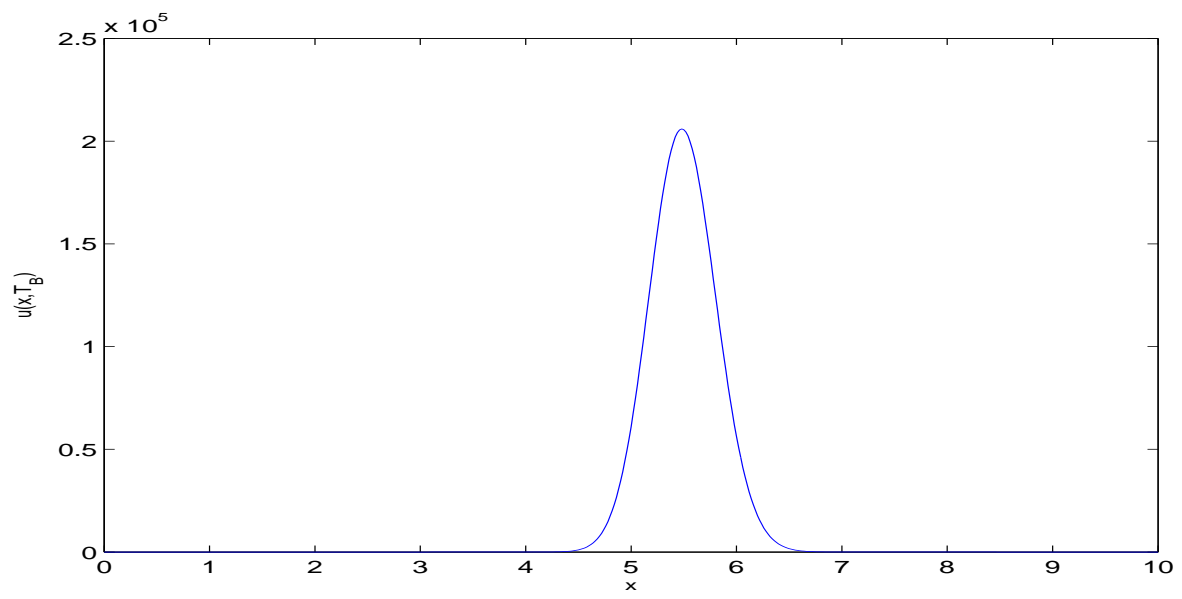

Figure 5: Plot of $u\left(x, T_{B}\right)$ as a function of the size $x$. 


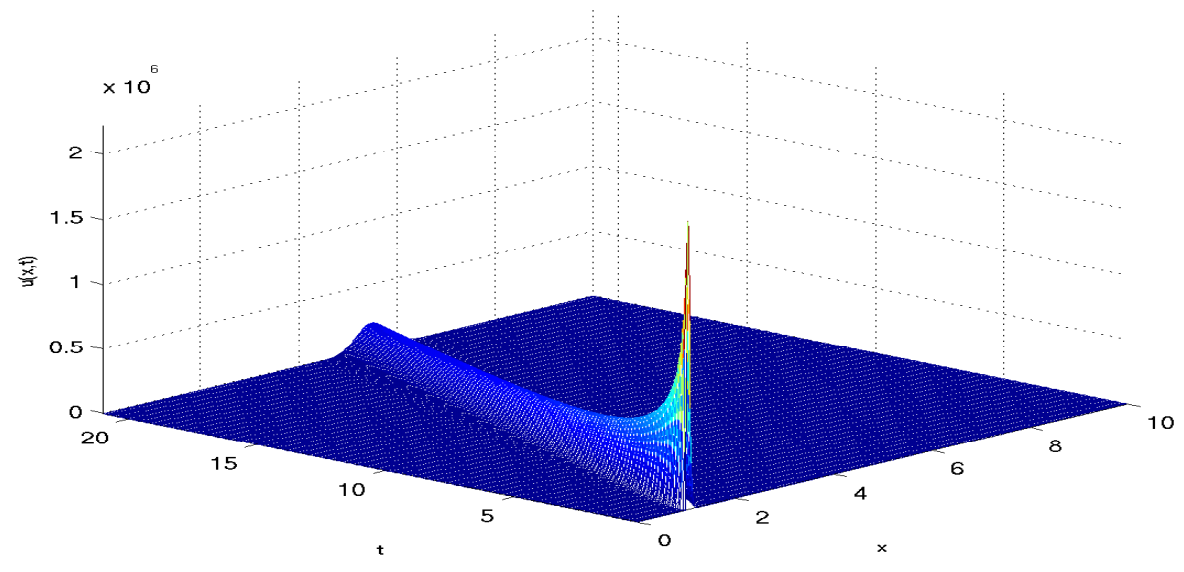

Figure 6: Plot of $u(x, t)$ as a function of size $x$ and time $t$.

\section{Vaccine Production Model}

In the vaccine production stage the shrimp are infected by distributing chopped dead shrimp infected with a recombinant virus evenly throughout the raceway. This transfected biomass is sufficiently large so that most of the shrimp can be infected in a short period of time, such as one day. There are other modes of transmission of virus in shrimp, such as cohabitation with infected shrimp that may be shedding the virus into the surrounding medium (waterborne infection). However, compared to the probability of shrimp becoming infected via ingestion, these modes of transmission can be assumed (reasonably for this investigation) to be negligible. Hence we will only assume infection via ingestion of dead transfected biomass. It is further assumed that all the shrimp have an equal chance of becoming infected by eating the infected biomass. The time interval considered here is 7 to 10 days. From [20] and [13] we know that during this time interval almost no shrimp progress into the chronic state. Therefore we only consider the following three compartment states: susceptible $(S)$, latently infected $(L)$ and acutely infected $(A)$ in our model.

In this model, we assume that shrimp will become instantly infected (i.e., progress into latent state) as soon as they ingest some of the infected biomass. As we have noted earlier, however, experimental observations suggest that there exists a temporal delay between the initial latent infection and initial acute infection [14]. Moreover, it is biologically unrealistic to expect all members of the shrimp population to progress into the acute phase at a fixed number of days after initial latent infection. In addition the shrimp in the acute phase have varying mortality rates because of the different times that they progress into the acute phase and also due to the differences in genetic make-up of the host. As we have already noted, we found that it is difficult to account for the class age history (i.e., the length of time that shrimp spend in a state) of shrimp in a particular (latent or acute) state using a system of delay PDE's with only size as the structure variable. This is because it is not obvious how to correctly represent the integral involving the delay. As an alternative, in order to model variable residency 
times we keep track of the class age and the size of shrimp by incorporating both size structure and class age structure into the latent and acute states.

We assume (based on experimental findings) that there is a possibility that shrimp can stay in each the latent and acute state for more than 7 to 10 days. Thus we can assume that the class age interval for both states is the same as the time interval $T_{V}$ that we consider in our model. Note that all shrimp from the biomass production raceway are healthy; there are no latently infected or acutely infected shrimp in the raceway at time $t=0$. We also know that shrimp in the acute state stop growing, which means that the growth rate in this state is $g=0$.

Based on the above discussions, our vaccine production model is given by

$$
\begin{aligned}
& S_{t}(x, t)+\left(g^{S}(x) S(x, t)\right)_{x}+m^{S}(x) S(x, t)=-\lambda S(x, t), \\
& L_{t}(x, t, \theta)+\left(g^{L}(x) L(x, t, \theta)\right)_{x}+L_{\theta}(x, t, \theta)+m^{L}(x) L(x, t, \theta)=-\gamma^{L}(\theta) L(x, t, \theta), \\
& A_{t}(x, t, \theta)+A_{\theta}(x, t, \theta)+m^{A}(\theta) A(x, t, \theta)=0, \\
& L(x, t, 0)=\lambda S(x, t), \\
& A(x, t, 0)=\int_{0}^{t} \gamma^{L}(\xi) L(x, t, \xi) d \xi, \\
& S(0, t)=0, \quad L(0, t, \theta)=0, \quad A(0, t, \theta)=0, \\
& S(x, 0)=S^{0}(x), \quad L(x, 0, \theta)=0, \quad A(x, 0, \theta)=0,
\end{aligned}
$$

where $(x, t, \theta) \in\left[x_{\min }, x_{\max }\right] \times\left[0, T_{V}\right] \times\left[0, T_{V}\right]$. In the above $S(x, t)$ denotes the density of individuals (number/unit mass) having mass $x$ at time $t$. The function $L(x, t, \theta)$ denotes the density of individuals (number/unit mass unit time) having mass $x$ at time $t$ that have spent $\theta$ days in the latent state, whereas the function $A(x, t, \theta)$ denotes the density of individuals (number/unit mass unit time) having mass $x$ at time $t$ that have spent $\theta$ days in the acute state. The quantity $g^{S}(x)$ denotes the growth rate of individuals (mass/unit time) in the susceptible state, $g^{L}(x)$ denotes the growth rate of individuals (mass/unit time) in the latent state. The function $m^{S}(x)$ denotes the mortality rate of individuals (1/unit time) in the susceptible state, and the function

$m^{L}(x)$ denotes the mortality rate ( 1 /unit time) of individuals in the latent state, and $m^{A}(\theta)$ denotes the mortality rate ( 1 /unit time) of the shrimp that spend $\theta$ days in the acute state. The latent to acute rate function $\gamma^{L}(\theta)$ denotes the rate $(1 /$ unit time) at which the shrimp in the latent state that have spent $\theta$ days in the latent state become acutely infected, while the quantity $\lambda$ denotes the infection rate (1/unit time) due to ingestion of chopped infected shrimp. Finally $S^{0}(x)$ denotes the initial population density of susceptible shrimp produced from the biomass production model.

\subsection{Relation to a Size Structured Epidemic Model}

In this section we show that the vaccine production model (4.1) can be reduced to a size structured three compartment epidemic model.

Let $\mathcal{L}(x, t)$ denote the density of individuals in the latent state having mass $x$ at 
time $t$. Noting that $L(x, 0, \theta)=0$, we have

$$
\mathcal{L}(x, t)=\int_{0}^{t} L(x, t, \theta) d \theta
$$

Integrating the second equation of (4.1) from 0 to $t$ with respect to $\theta$, we have

$$
\begin{aligned}
& \int_{0}^{t} L_{t}(x, t, \theta) d \theta+\int_{0}^{t}\left(g^{L}(x) L(x, t, \theta)\right)_{x} d \theta+\int_{0}^{t} L_{\theta}(x, t, \theta) d \theta+\int_{0}^{t} m^{L}(x) L(x, t, \theta) d \theta \\
& =-\int_{0}^{t} \gamma^{L}(\theta) L(x, t, \theta) d \theta .
\end{aligned}
$$

Simplifying we find

$$
\begin{aligned}
& \int_{0}^{t} L_{t}(x, t, \theta) d \theta+\left(g^{L}(x) \mathcal{L}(x, t)\right)_{x}+L(x, t, t)-L(x, t, 0)+m^{L}(x) \mathcal{L}(x, t) \\
& =-\int_{0}^{t} \gamma^{L}(\theta) L(x, t, \theta) d \theta .
\end{aligned}
$$

Since

$$
\mathcal{L}_{t}(x, t)=\frac{d \int_{0}^{t} L(x, t, \theta) d \theta}{d t}=L(x, t, t)+\int_{0}^{t} L_{t}(x, t, \theta) d \theta
$$

and $L(x, t, 0)=\lambda S(x, t)$, we obtain

$$
\mathcal{L}_{t}(x, t)+\left(g^{L}(x) \mathcal{L}(x, t)\right)_{x}+m^{L}(x) \mathcal{L}(x, t)=\lambda S(x, t)-\int_{0}^{t} \gamma^{L}(\theta) L(x, t, \theta) d \theta .
$$

If we define the average

$$
\Gamma^{L}(x, t)=\frac{\int_{0}^{t} \gamma^{L}(\theta) L(x, t, \theta) d \theta}{\int_{0}^{t} L(x, t, \theta) d \theta}
$$

then on substituting (4.4) in (4.3) we obtain the equation

$$
\mathcal{L}_{t}(x, t)+\left(g^{L}(x) \mathcal{L}(x, t)\right)_{x}+m^{L}(x) \mathcal{L}(x, t)=\lambda S(x, t)-\Gamma^{L}(x, t) \mathcal{L}(x, t) .
$$

Next we let $\mathcal{A}(x, t)$ denote the density of individuals in the acute state having size $x$ at time $t$. Noting that $A(x, 0, \theta)=0$, we have

$$
\mathcal{A}(x, t)=\int_{0}^{t} A(x, t, \theta) d \theta
$$

Integrating the third equation of (4.1) from 0 to $t$ with respect to $\theta$, we have

$$
\int_{0}^{t} A_{t}(x, t, \theta) d \theta+\int_{0}^{t} A_{\theta}(x, t, \theta) d \theta+\int_{0}^{t} m^{A}(\theta) A(x, t, \theta) d \theta=0 .
$$

Simplifying we have

$$
\int_{0}^{t} A_{t}(x, t, \theta) d \theta+A(x, t, t)-A(x, t, 0)+\int_{0}^{t} m^{A}(\theta) A(x, t, \theta) d \theta=0 .
$$


Since

$$
\mathcal{A}_{t}(x, t)=\frac{d \int_{0}^{t} A(x, t, \theta) d \theta}{d t}=A(x, t, t)+\int_{0}^{t} A_{t}(x, t, \theta) d \theta
$$

and

$$
A(x, t, 0)=\int_{0}^{t} \gamma^{L}(\theta) L(x, t, \theta) d \theta
$$

we obtain

$$
\mathcal{A}_{t}(x, t)+\int_{0}^{t} m^{A}(\theta) A(x, t, \theta) d \theta=\int_{0}^{t} \gamma^{L}(\theta) L(x, t, \theta) d \theta .
$$

As in the case for the latent state, we define the average

$$
M^{A}(x, t)=\frac{\int_{0}^{t} m^{A}(\theta) A(x, t, \theta) d \theta}{\int_{0}^{t} A(x, t, \theta) d \theta} .
$$

Then on substituting (4.8) in (4.7) we obtain the equation

$$
\mathcal{A}_{t}(x, t)+M^{A}(x, t) \mathcal{A}(x, t)=\Gamma^{L}(x, t) \mathcal{L}(x, t),
$$

Thus, by summing over the class age the vaccine production model (4.1) reduces to

$$
\begin{aligned}
& S_{t}(x, t)+\left(g^{S}(x) S(x, t)\right)_{x}+m^{S}(x) S(x, t)=-\lambda S(x, t), \\
& \mathcal{L}_{t}(x, t)+\left(g^{L}(x) \mathcal{L}(x, t)\right)_{x}+m^{L}(x) \mathcal{L}(x, t)=\lambda S(x, t)-\Gamma^{L}(x, t) \mathcal{L}(x, t), \\
& \mathcal{A}_{t}(x, t)+M^{A}(x, t) \mathcal{A}(x, t)=\Gamma^{L}(x, t) \mathcal{L}(x, t), \\
& S(0, t)=0, \quad \mathcal{L}(0, t)=0, \quad \mathcal{A}(0, t)=0, \\
& S(x, 0)=S^{0}(x), \quad \mathcal{L}(x, 0)=0, \quad \mathcal{A}(x, 0)=0,
\end{aligned}
$$

with $\Gamma^{L}$ and $M^{A}$ as defined in (4.4) and (4.8) respectively. The model (4.10) is a three compartment size structured model which involves only one structure variable, namely the mass $x$.

\subsection{Method of Characteristics for the Vaccine Production Model}

In this section we use the method of characteristics to obtain the solution of (4.1). Consider the first equation of (4.1). The characteristic equations in this case are as follows:

$$
\begin{aligned}
& \frac{d \tilde{t}(s)}{d s}=1, \\
& \tilde{t}(0)=t
\end{aligned}
$$

which implies $\tilde{t}(s)=t+s$, and

$$
\begin{aligned}
& \frac{d \tilde{x}(s)}{d s}=g^{S}(\tilde{x}(s)), \\
& \tilde{x}(0)=x,
\end{aligned}
$$


which implies that $\int_{0}^{s} \frac{1}{g^{S}(\tilde{x}(\tau))} d \tilde{x}(\tau)=s$. Let $y=\tilde{x}(\tau)$, so that we have

$$
\exp \left(\int_{x}^{\tilde{x}(s)} \frac{1}{g^{S}(y)} d y\right)=\exp (s)
$$

which implies that

$$
\exp \left(\int_{\tilde{x}(s)}^{x_{\max }} \frac{1}{g^{S}(y)} d y-\int_{x}^{x_{\max }} \frac{1}{g^{S}(y)} d y\right)=\exp (-s) .
$$

Let $h^{S}(r)=\exp \left(\int_{r}^{x_{\max }} \frac{1}{g^{S}(y)} d y\right)$, then $h^{S}(r)$ is a decreasing function. Hence its inverse function which we denote by $\left(h^{S}\right)^{-1}$ exists. By (4.11), we have that

$$
h^{S}(\tilde{x}(s))=h^{S}(x) \exp (-s),
$$

which implies that

$$
\tilde{x}(s)=\left(h^{S}\right)^{-1}\left(h^{S}(x) \exp (-s)\right) .
$$

We now consider the solution of $S(x, t)$ along the characteristic curve. We then have

$$
\begin{aligned}
& \frac{d S(\tilde{x}(s), \tilde{t}(s))}{d s}=-\left(g_{x}^{S}(\tilde{x}(s))+m^{S}(\tilde{x}(s))+\lambda\right) S(\tilde{x}(s), \tilde{t}(s)), \\
& S(\tilde{x}(0), \tilde{t}(0))=S(x, t),
\end{aligned}
$$

which implies that

$$
S(x, t)=S(\tilde{x}(s), \tilde{t}(s)) \exp \left(\int_{0}^{s}\left(g_{x}^{S}(\tilde{x}(\tau))+m^{S}(\tilde{x}(\tau))+\lambda\right) d \tau\right) .
$$

Let $y=\tilde{x}(\tau)$ so that $y=\left(h^{S}\right)^{-1}\left(h^{S}(x) \exp (-\tau)\right)$. We then find that $\tau=\ln \left(\frac{h^{S}(x)}{h^{S}(y)}\right)$, which implies that

$$
d \tau=-\frac{\left(h^{S}\right)^{\prime}(y)}{h^{S}(y)} d y
$$

Noting that $h^{S}(y)=\exp \left(\int_{y}^{x_{\max }} \frac{1}{g^{S}(\tau)} d \tau\right)$, we find that

$$
\left(h^{S}\right)^{\prime}(y)=-\frac{h^{S}(y)}{g^{S}(y)}
$$

By (4.14) and (4.15), we have $d \tau=\frac{1}{g^{S}(y)} d y$. Thus simple calculations yield that

$$
\begin{aligned}
\exp \left(\int_{0}^{s}\left(g_{x}^{S}(\tilde{x}(\tau))+m^{S}(\tilde{x}(\tau)) d \tau\right)\right. & =\exp \left(\int_{x}^{\tilde{x}(s)} \frac{g_{y}^{S}(y)+m^{S}(y)}{g^{S}(y)} d y\right) \\
& =\frac{g^{S}(\tilde{x}(s))}{g^{S}(x)} \exp \left(\int_{x}^{\tilde{x}(s)} \frac{m^{S}(y)}{g^{S}(y)} d y\right)
\end{aligned}
$$


Hence from (4.13), we obtain

$$
S(x, t)=\frac{g^{S}(\tilde{x}(s))}{g^{S}(x)} S(\tilde{x}(s), \tilde{t}(s)) \exp \left(-\int_{\tilde{x}(s)}^{x} \frac{m^{S}(y)}{g^{S}(y)} d y\right) \exp (\lambda s) .
$$

Thus, if $t \leq \int_{x_{\min }}^{x} \frac{1}{g^{S}(y)} d y$, we have

$$
S(x, t)=\frac{g^{S}(\tilde{x}(-t))}{g^{S}(x)} S^{0}(\tilde{x}(-t)) \exp \left(-\int_{\tilde{x}(-t)}^{x} \frac{m^{S}(y)}{g^{S}(y)} d y\right) \exp (-\lambda t)
$$

and if $t>\int_{x_{\min }}^{x} \frac{1}{g^{S}(y)} d y$, we have

$$
S(x, t)=0
$$

We then consider the second equation of (4.1). As above we consider the characteristic equations

$$
\begin{aligned}
& \tilde{t}(s)=t+s, \tilde{\theta}(s)=\theta+s \\
& \tilde{x}(s)=\left(h^{L}\right)^{-1}\left(h^{L}(x) \exp (-s)\right), \text { where } h^{L}(r)=\exp \left(\int_{r}^{x_{\max }} \frac{1}{g^{L}(y)} d y\right) .
\end{aligned}
$$

We now consider the solution $L(x, t, \theta)$ along the characteristic curve,

$$
\begin{aligned}
& \frac{d L(\tilde{x}(s), \tilde{t}(s), \tilde{\theta}(s))}{d s}=-\left\{g_{x}^{L}(\tilde{x}(s))+m^{L}(\tilde{x}(s))+\gamma^{L}(\tilde{\theta}(s))\right\} L(\tilde{x}(s), \tilde{t}(s), \tilde{\theta}(s)) \\
& L(\tilde{x}(s), \tilde{t}(s), \tilde{\theta}(s))=L(x, t, \theta)
\end{aligned}
$$

which implies that

$L(x, t, \theta)=\frac{g^{L}(\tilde{x}(s))}{g^{L}(x)} L(\tilde{x}(s), \tilde{t}(s), \tilde{\theta}(s)) \exp \left(-\int_{\tilde{x}(s)}^{x} \frac{m^{L}(y)}{g^{L}(y)} d y\right) \exp \left(-\int_{\tilde{\theta}(s)}^{\theta} \gamma^{L}(y) d y\right)$.

Note that $\tilde{\theta}(s) \leq \tilde{t}(s)$ for every $s \in \mathbb{R}$, and we have that if $\theta \leq \int_{x_{\min }}^{x} \frac{1}{g^{L}(y)} d y$, then

$L(x, t, \theta)=\lambda S(\tilde{x}(-\theta), t-\theta) \frac{g^{L}(\tilde{x}(-\theta))}{g^{L}(x)} \exp \left(-\int_{\tilde{x}(-\theta)}^{x} \frac{m^{L}(y)}{g^{L}(y)} d y\right) \exp \left(-\int_{0}^{\theta} \gamma^{L}(y) d y\right)$,

and if $\theta>\int_{x_{\min }}^{x} \frac{1}{g^{L}(y)} d y$, then we have

$$
L(x, t, \theta)=0
$$

Finally, we consider the third equation of (4.1). The characteristic equations in this case are

$$
\tilde{t}(s)=t+s, \tilde{\theta}(s)=\theta+s, \tilde{x}(s)=x .
$$


We now consider the solution $A(x, t, \theta)$ along the characteristic curve and find

$$
\begin{aligned}
& \frac{d A(\tilde{x}(s), \tilde{t}(s), \tilde{\theta}(s))}{d s}=-m^{A}(\tilde{\theta}(s)) A(\tilde{x}(s), \tilde{t}(s), \tilde{\theta}(s)) \\
& A(\tilde{x}(s), \tilde{t}(s), \tilde{\theta}(s))=A(x, t, \theta),
\end{aligned}
$$

which implies that

$$
A(x, t, \theta)=A(\tilde{x}(s), \tilde{t}(s), \tilde{\theta}(s)) \exp \left(-\int_{\tilde{\theta}(s)}^{\theta} m^{A}(y) d y\right) .
$$

Note that $\tilde{\theta}(s) \leq \tilde{t}(s)$ for every $s \in \mathbb{R}$, and we have that if $x>x_{\min }$

$$
A(x, t, \theta)=\exp \left(-\int_{0}^{\theta} m^{A}(y) d y\right) \int_{0}^{t-\theta} \gamma^{L}(y) L(x, t-\theta, y) d y
$$

and if $x=x_{\min }$, we have

$$
A(x, t, \theta)=0
$$

\subsection{Numerical Scheme for the Vaccine Production Model}

We partition the $x$ interval $\left[x_{\min }, x_{\max }\right]$ with $n_{x}+1$ discrete points $x_{j}=j \Delta x$ with $\Delta x=\frac{x_{\max }-x_{\min }}{n_{x}}$ and $j=0,1, \ldots, n_{x}$. We divide the $t$ interval $\left[0, T_{V}\right]$ into $n_{t}+1$ discrete points $t_{k}=k \Delta t$ with $\Delta t=T_{V} / n_{t}$ and $k=0,1, \ldots, n_{t}$. Finally we also divide the $\theta$ interval $\left[0, T_{V}\right]$ into $n_{\theta}+1$ discrete points $\theta_{l}=l \Delta \theta$ with $\Delta \theta=T_{V} / n_{\theta}$ and $l=0,1, \ldots, n_{\theta}$. We denote the finite difference approximations of $S\left(x_{j}, t_{k}\right), L\left(x_{j}, t_{k}, \theta_{l}\right)$ and $A\left(x_{j}, t_{k}, \theta_{l}\right)$ by

$$
\begin{aligned}
& S_{j}^{k} \approx S\left(x_{j}, t_{k}\right), \\
& L_{j}^{k, l} \approx L\left(x_{j}, t_{k}, \theta_{l}\right), \\
& A_{j}^{k, l} \approx A\left(x_{j}, t_{k}, \theta_{l}\right),
\end{aligned}
$$

and we let

$$
\begin{aligned}
& g_{j}^{S}=g^{S}\left(x_{j}\right), g_{j}^{L}=g^{L}\left(x_{j}\right), \\
& m_{j}^{S}=m^{S}\left(x_{j}\right), m_{j}^{L}=m^{L}\left(x_{j}\right) \\
& \gamma^{L, l}=\gamma^{L}\left(\theta_{l}\right), m^{A, l}=m^{A}\left(\theta_{l}\right) .
\end{aligned}
$$

We discretize the vaccine production model (4.1) using an implicit scheme as follows. 
For $j=1,2, \ldots n_{x}, k=0,1, \ldots n_{t}$, and $l=0,1, \ldots, n_{\theta}$ we have

$$
\frac{S_{j}^{k+1}-S_{j}^{k}}{\Delta t}+\frac{g_{j}^{S} S_{j}^{k+1}-g_{j-1}^{S} S_{j-1}^{k+1}}{\Delta x}+m_{j}^{S} S_{j}^{k+1}=-\lambda S_{j}^{k+1}
$$

(ii)

$$
\begin{gathered}
\text { (ii) } \frac{L_{j}^{k+1, l+1}-L_{j}^{k, l+1}}{\Delta t}+\frac{g_{j}^{L} L_{j}^{k+1, l+1}-g_{j-1}^{L} L_{j-1}^{k+1, l+1}}{\Delta x}+\frac{L_{j}^{k+1, l+1}-L_{j}^{k+1, l}}{\Delta \theta} \\
\quad+m_{j}^{L} L_{j}^{k+1, l+1}=-\gamma^{L, l+1} L_{j}^{k+1, l+1}, \\
\text { (iii) } \frac{A_{j}^{k+1, l+1}-A_{j}^{k, l+1}}{\Delta t}+\frac{A_{j}^{k+1, l+1}-A_{j}^{k+1, l}}{\Delta \theta}+m^{A, l+1} A_{j}^{k+1, l+1}=0,
\end{gathered}
$$

(iv) $S_{0}^{k+1}=0$,

(v) $L_{0}^{k+1, l+1}=0, L_{j}^{0, l+1}=0, L_{j}^{k+1,0}=\lambda S_{j}^{k+1}$,

(vi) $A_{0}^{k+1, l+1}=0, A_{j}^{0, l+1}=0, A_{j}^{k+1,0}=\sum_{l=1}^{\tilde{n}_{\theta, k+1}} \gamma^{L, l} L_{j}^{k+1, l} \Delta \theta$, where $\tilde{n}_{\theta, k}=\left[t_{k} / \Delta \theta\right]$

with $[z]$ being the usual greatest integer part of $\mathrm{z}$.

From the above scheme we note that the solution remains positive for all time. This is important from the biological point of view.

Since $\mathcal{L}(x, t)=\int_{0}^{t} L(x, t, \theta) d \theta$ and $\mathcal{A}(x, t)=\int_{0}^{t} A(x, t, \theta) d \theta$, from the solution of (4.24) we have $\mathcal{L}_{j}^{k}=\sum_{l=1}^{\tilde{n}_{\theta, k}} L_{j}^{k, l} \Delta \theta$ and $\mathcal{A}_{j}^{k}=\sum_{l=1}^{\tilde{n}_{\theta, k}} A_{j}^{k, l} \Delta \theta$.

Let $B^{S}, B^{L}$ and $B^{A}$ denote the biomass of the susceptible, latent and acute populations, respectively. Then

$$
B^{S}(t)=\int_{x_{\min }}^{x_{\max }} x S(x, t) d x, B^{L}(t)=\int_{x_{\min }}^{x_{\max }} x \mathcal{L}(x, t) d x, \text { and } B^{A}(t)=\int_{x_{\min }}^{x_{\max }} x \mathcal{A}(x, t) d x .
$$

The corresponding ratios of the biomass in each state to the total biomass are denoted by

$$
\begin{aligned}
\operatorname{BRatio}_{S}(t) & =\frac{B^{S}(t)}{B^{S}(t)+B^{L}(t)+B^{A}(t)}, \\
\operatorname{BRatio}_{L}(t) & =\frac{B^{L}(t)}{B^{S}(t)+B^{L}(t)+B^{A}(t)}, \\
\operatorname{BRatio}_{A}(t) & =\frac{B^{A}(t)}{B^{S}(t)+B^{L}(t)+B^{A}(t)} .
\end{aligned}
$$

We also let $N^{S}, N^{L}$ and $N^{A}$ denote the total number in the susceptible, latent and acute populations. Then

$$
N^{S}(t)=\int_{x_{\min }}^{x_{\max }} S(x, t) d x, N^{L}(t)=\int_{x_{\min }}^{x_{\max }} \mathcal{L}(x, t) d x, \text { and } N^{A}(t)=\int_{x_{\min }}^{x_{\max }} \mathcal{A}(x, t) d x .
$$


The corresponding ratios of the total number in each state to the total population are defined as

$$
\begin{aligned}
& \operatorname{NRatio}_{S}(t)=\frac{N^{S}(t)}{N^{S}(t)+N^{L}(t)+N^{A}(t)}, \\
& \operatorname{NRatio}_{L}(t)=\frac{N^{L}(t)}{N^{S}(t)+N^{L}(t)+N^{A}(t)}, \\
& \operatorname{NRatio}_{A}(t)=\frac{N^{A}(t)}{N^{S}(t)+N^{L}(t)+N^{A}(t)} .
\end{aligned}
$$

\section{Numerical Simulations for the Coupled Bio- mass and Vaccine Model}

We simulate the biomass production model over 3 weeks. The results of this model are used as initial conditions to the vaccine production model. In the following examples, we assume that $x_{\min }=0 \mathrm{gm}, x_{\max }=10 \mathrm{gm}, T_{V}=7$ days. The mesh size for $\Delta x$, $\Delta t$ and $\Delta \theta$ are given by $0.02,0.01$ and 0.001 , respectively. Some of the parameters in (4.1) are defined by $\lambda=1.5, g^{S}(x)=0.214, g^{L}(x)=0.107, m^{S}(x)=0.0014, m^{L}(x)=$ $0.0028, m^{A}(\theta)=1.21 /\left[1.21+(\theta-3)^{2}\right]$.

The mortality function $m^{A}(\theta)$ is plotted along with the cumulative mortality in Figure 7 . The cumulative mortality captures the effect of progression of TSV infection on the mortality rate for acutely infected shrimp. With this mortality function we perform four different numerical simulations each with different choices of the latent to acute rate function. We present the resulting plots of population ratios corresponding to these choices of latent to acute rate functions to illustrate how this quantity affects the population ratios and hence the dynamics of the epidemic.
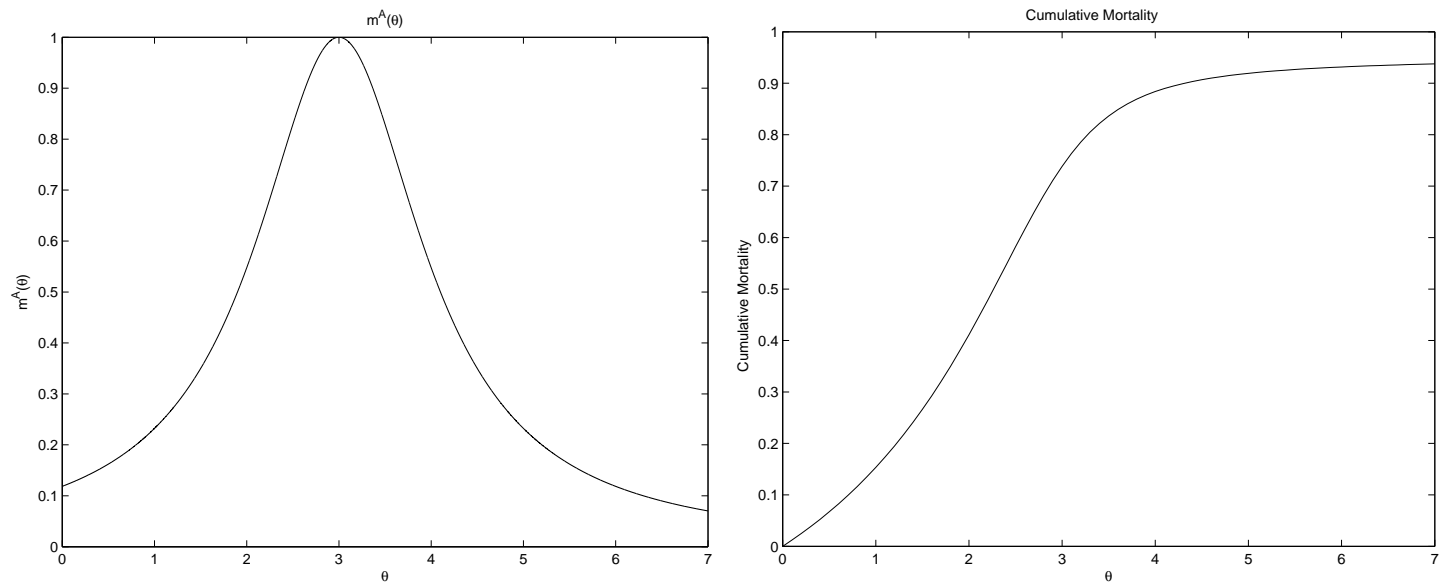

Figure 7: (left) The mortality function $m^{A}(\theta)$. (right) Cumulative mortality $1-\exp \left(-\int_{0}^{\theta} m^{A}(\tau) d \tau\right)$ 


\subsection{Example 1}

In this section the latent to acute rate function is taken as

$$
\gamma^{L}(\theta)=\gamma_{1}^{L}(\theta)=\frac{1}{1+\exp (-4(\theta-3))}
$$

In Figure 8 we plot $\gamma_{1}^{L}(\theta)$ (left) and the cumulative latent to acute function (right)
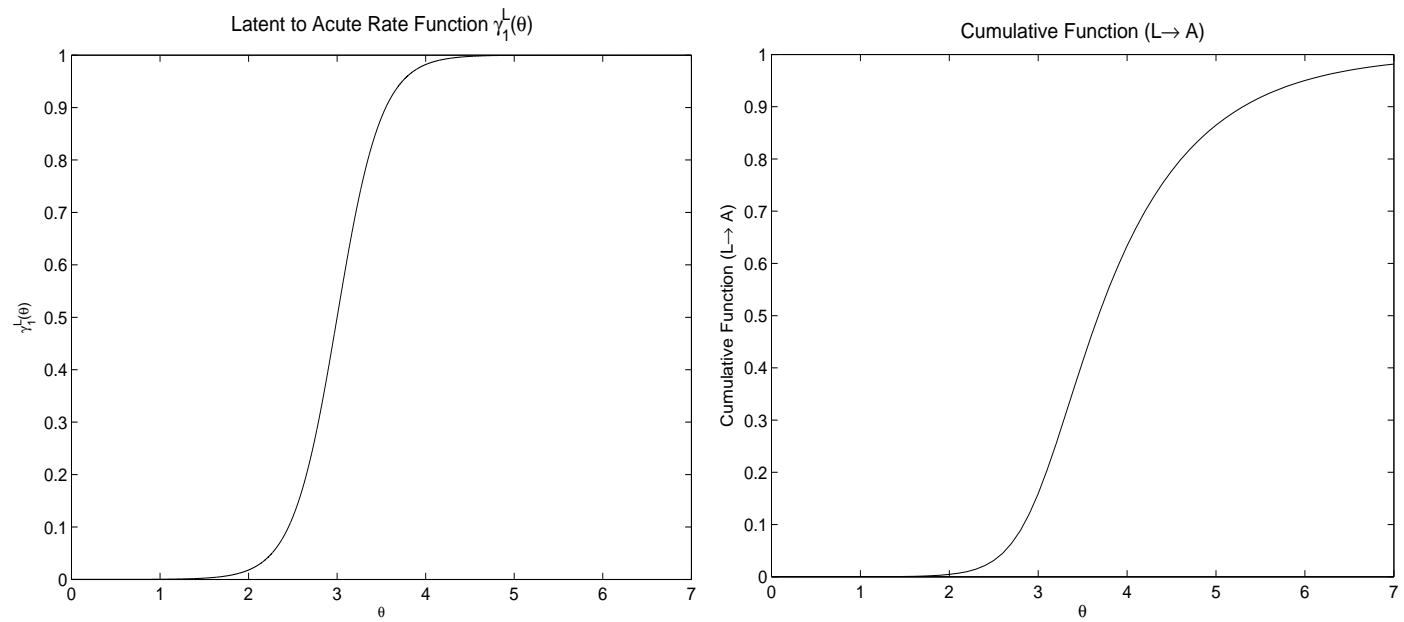

Figure 8: (left) $\gamma^{L}(\theta),\left(\right.$ right) $1-\exp \left(-\int_{0}^{\theta} \gamma^{L}(\tau) d \tau\right)$

which is given to be $1-\exp \left(-\int_{0}^{\theta} \gamma_{1}^{L}(\tau) d \tau\right)$. In this figure we observe that after 2 days a non-negligible portion of latently infected shrimp start becoming acute. About $2 \%$ shrimp stay latently infected for more than 7 days. In Figure 9 we plot the total number, $N^{S}, N^{L}$ and $N^{A}$ of the susceptible, latent, and acute populations, respectively in the top left hand corner. We plot the total biomass, $B^{S}, B^{L}$ and $B^{A}$ in the top right hand corner. In the bottom left hand corner we plot the NRatios for all three populations, and in the bottom right hand corner we plot the respective BRatios. All of the quantities plotted are defined in Section 4.3. From this figure we see that almost all susceptible shrimp become latently infected after 2 days. The maximum number of latently infected shrimp is seen at about 2.5 days. We observe that the acute phase starts after 2 days. At day 7 , about $89 \%$ of shrimp in the raceway are acutely infected. 

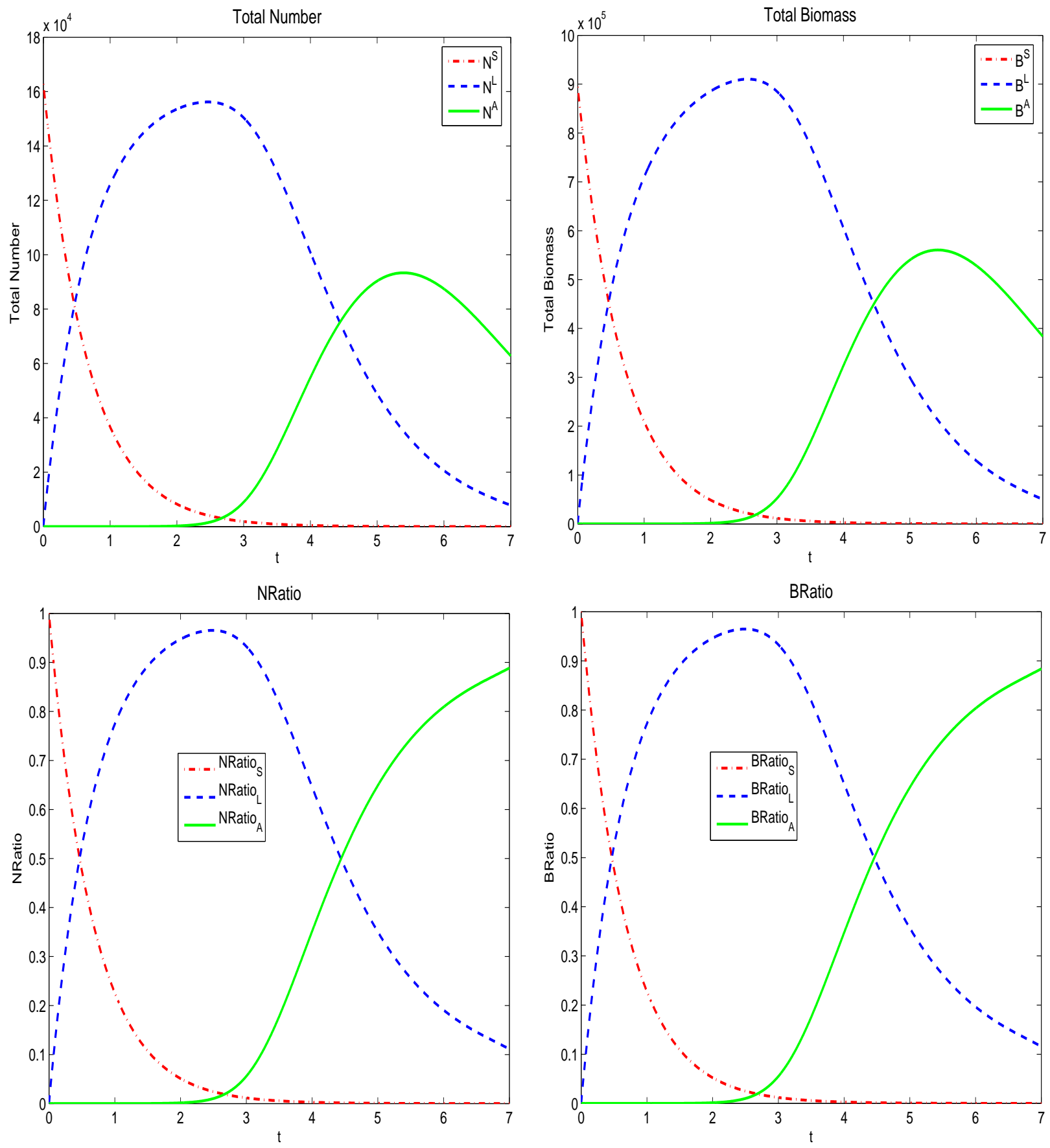

Figure 9: Results for the susceptible, latent and acute populations, with $\gamma^{L}(\theta)=\gamma_{1}^{L}(\theta)$. 


\subsection{Example 2}

In this section the latent to acute rate function is taken as

$$
\gamma^{L}(\theta)=\gamma_{2}^{L}(\theta)=\frac{10}{1+\exp (-4(\theta-3))} .
$$

In Figure 10 we plot $\gamma_{2}^{L}(\theta)$ (left) and the cumulative latent to acute function (right)


Figure 10: (left) $\gamma^{L}(\theta),($ right $) 1-\exp \left(-\int_{0}^{\theta} \gamma^{L}(\tau) d \tau\right)$

which in this case is given to be $1-\exp \left(-\int_{0}^{\theta} \gamma_{2}^{L}(\tau) d \tau\right)$. In this figure we observe that around 2 days some portion of latently infected shrimp start becoming acute. A negligible number of shrimp stay latently infected for more than 4 days. As done in example 1, in Figure 11 we plot the total number, total biomass, NRatios and BRatios of the susceptible, latent and acute populations. We observe that almost all susceptible shrimp become latently infected after 2 days. The maximum number of latently infected shrimp occurs at about 2 days. We observe that the acute phase starts around 2 days. At day 7 , about $99 \%$ of shrimp in the raceway are acutely infected. 



Figure 11: Results for the susceptible, latent and acute populations, with $\gamma^{L}(\theta)=\gamma_{2}^{L}(\theta)$. 


\subsection{Example 3}

In this section the latent to acute rate function is taken as

$$
\gamma^{L}(\theta)=\gamma_{3}^{L}(\theta)=\frac{1}{1+\exp (-5(\theta-4))} .
$$

In Figure 12 we plot $\gamma_{3}^{L}(\theta)$ (left) and the cumulative latent to acute rate function
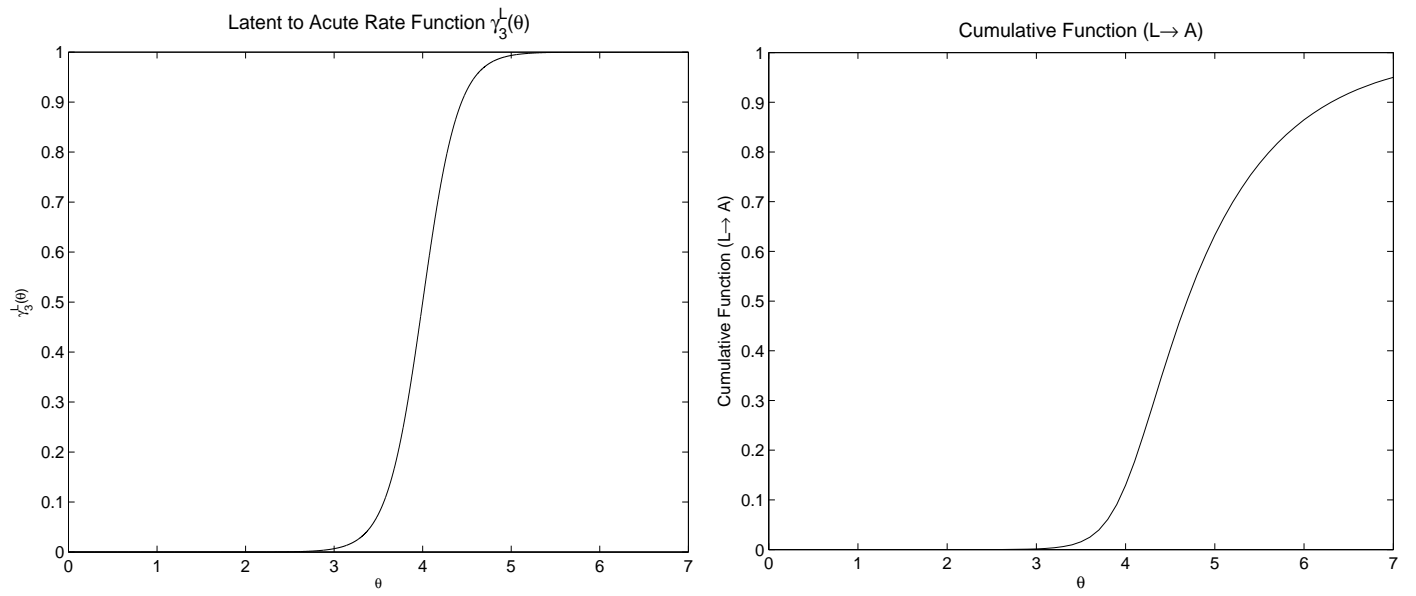

Figure 12: (left) $\gamma^{L}(\theta),($ right $) 1-\exp \left(-\int_{0}^{\theta} \gamma^{L}(\tau) d \tau\right)$

(right) given as $1-\exp \left(-\int_{0}^{\theta} \gamma_{3}^{L}(\tau) d \tau\right)$. We observe that after 3 days some portion of latently infected shrimp start becoming acute. About 5\% shrimp stay latently infected for more than 7 days. In Figure 13 we plot the total number, total biomass, NRatios and BRatios for the susceptible, latent and acute populations. We see that almost all susceptible shrimp become latently infected after 2 days. The maximum number of latently infected shrimp occurs at about 3.5 days. We observe that the acute phase starts after 3 days. At day 7 , about $82 \%$ of shrimp in the raceway are acutely infected. 

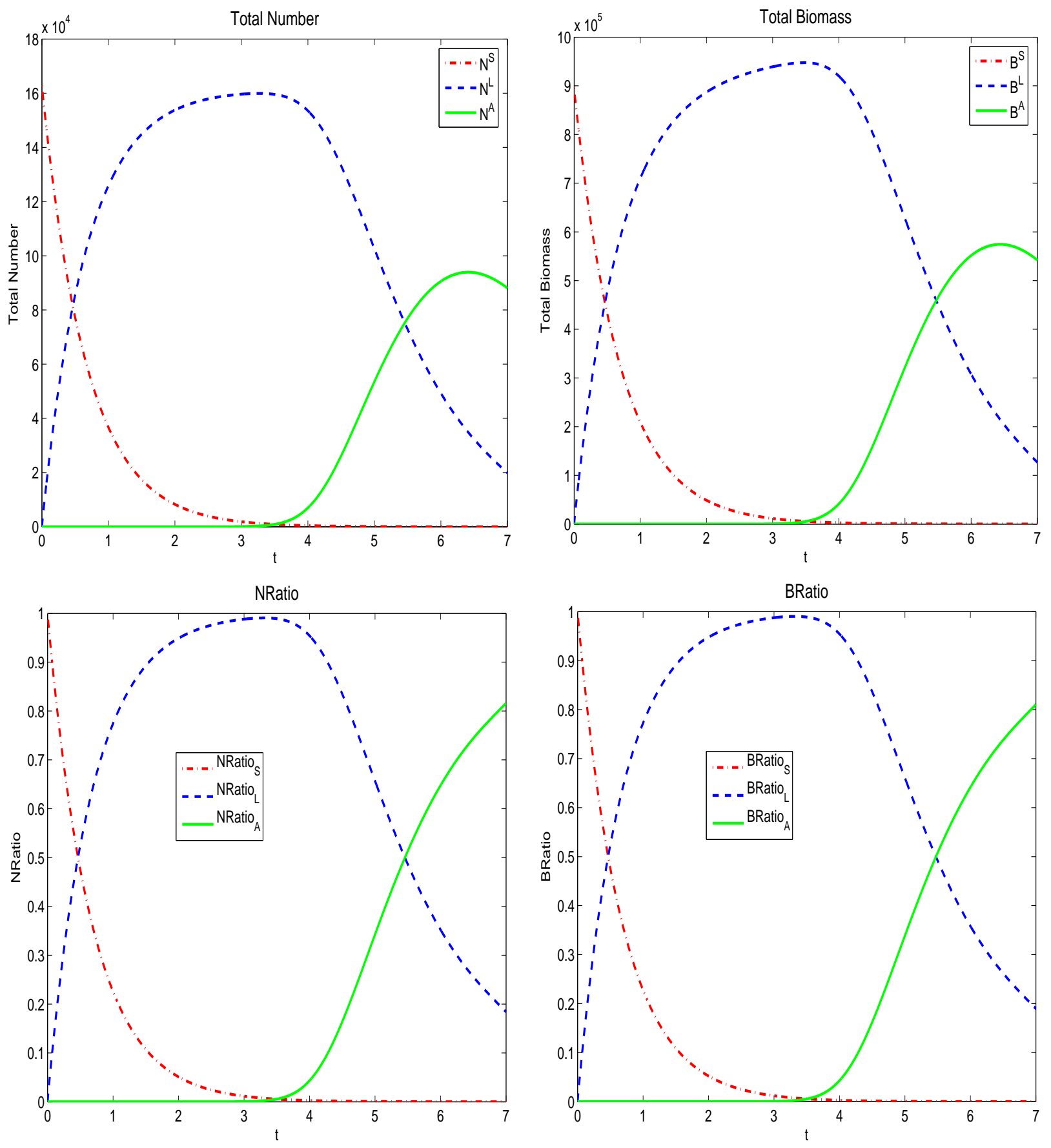

Figure 13: Results for the susceptible, latent, and acute populations with $\gamma^{L}(\theta)=\gamma_{3}^{L}(\theta)$. 


\subsection{Example 4}

In this section the latent to acute rate function is taken as

$$
\gamma^{L}(\theta)=\gamma_{4}^{L}(\theta)=\frac{10}{1+\exp (-5(\theta-4))} .
$$

In Figure 14 we plot $\gamma_{4}^{L}(\theta)$ (left) and the cumulative latent to acute function (right)
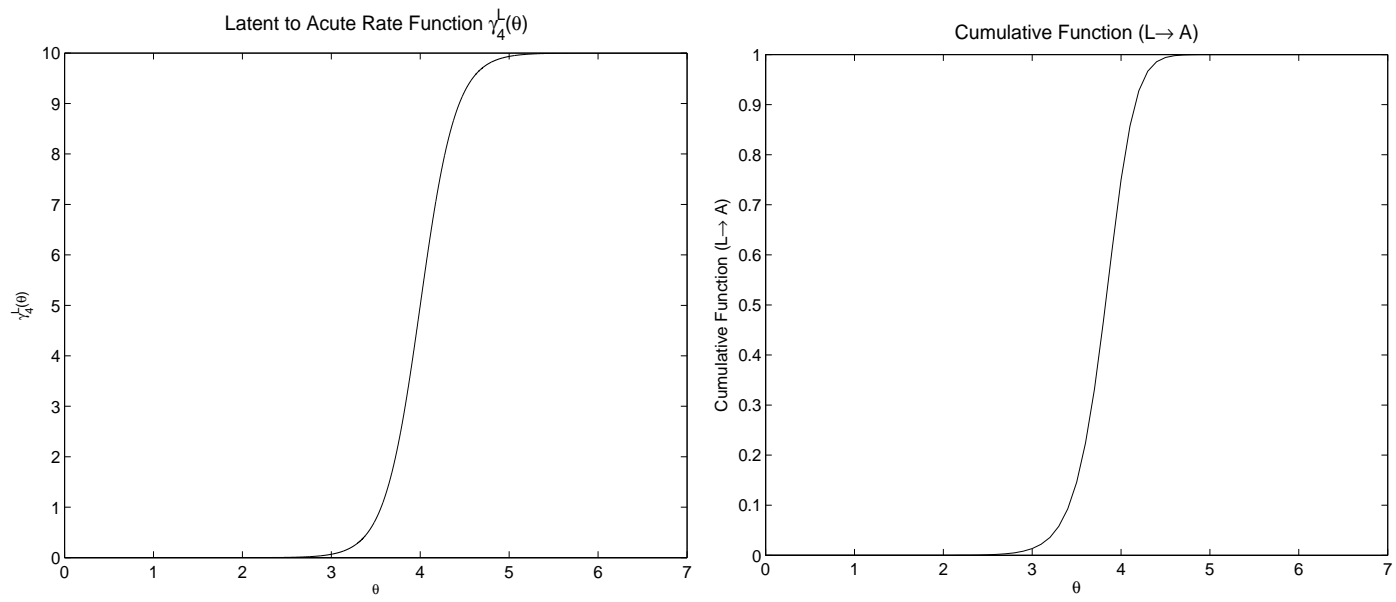

Figure 14: (left) $\gamma^{L}(\theta)$, (right) $1-\exp \left(-\int_{0}^{\theta} \gamma^{L}(\tau) d \tau\right)$

given to be $1-\exp \left(-\int_{0}^{\theta} \gamma_{4}^{L}(\tau) d \tau\right)$. In this figure we observe that around 3 days some portion of latently infected shrimp start becoming acute. A negligible number of shrimp stay latently infected for more than 5 days. In Figure 15 we plot the total number, total biomass, NRatios and BRatios for the susceptible, latent and acute populations as done in the previous examples. Here we see that almost all susceptible shrimp become latently infected after 2 days. The maximum number of latently infected shrimp occurs at about 3 days. We observe that the acute phase starts around 3 days. At day 7 , about $98 \%$ of shrimp in the raceway are acutely infected. 



Figure 15: Results for the susceptible, latent, and acute populations with $\gamma^{L}(\theta)=\gamma_{4}^{L}(\theta)$. 


\subsection{Example Summary}

In this section we compare the results presented in examples 1, 2, 3 and 4, to determine the effect that the latent to acute rate function, $\gamma^{L}(\theta)$, has on the dynamics of the epidemic. In Figure 16, we plot the four different cumulative latent to acute functions that were used in the different examples presented earlier. In Figures 17, and 18, we plot the total number, total biomass, NRatios and BRatios for the simulations performed with $\gamma_{1}^{L}, \gamma_{2}^{L}, \gamma_{3}^{L}$, and $\gamma_{4}^{L}$ for the latent population, and acute population respectively.

In Figure 17 we note that the time of occurence of the peak of each curve corresponding to total number or total biomass, as well as the maximum amplitude of each curve are determined by the shape of the corresponding function $\gamma^{L}$. In particular, the determining factors are the time when a non-negligible number of latently infected shrimp start becoming acute, and the rate at which the latent population becomes acute as seen in Figure 16.

In Figure 18 we note that the time of occurence of the peak of each curve corresponding to total number or total biomass is determined by the time when a non-negligible number of latently infected shrimp start becoming acute, and the rate at which the latent population becomes acute (see Figure 16). The rate at which the population becomes acute and the mortality rate (see Figure 7) also determine the maximum amplitude of each curve. For the acute population the mortality rate, $m^{A}$, depends on how long the shrimp stay in the acute phase and thus plays an important role in the dynamics of this population.

In conclusion, the latent to acute rate function $\gamma^{L}$ plays a very important role in determining the particular shape of the curves corresponding to the total number, total biomass, NRatio and BRatio, i.e., the progression of the viral epidemic.

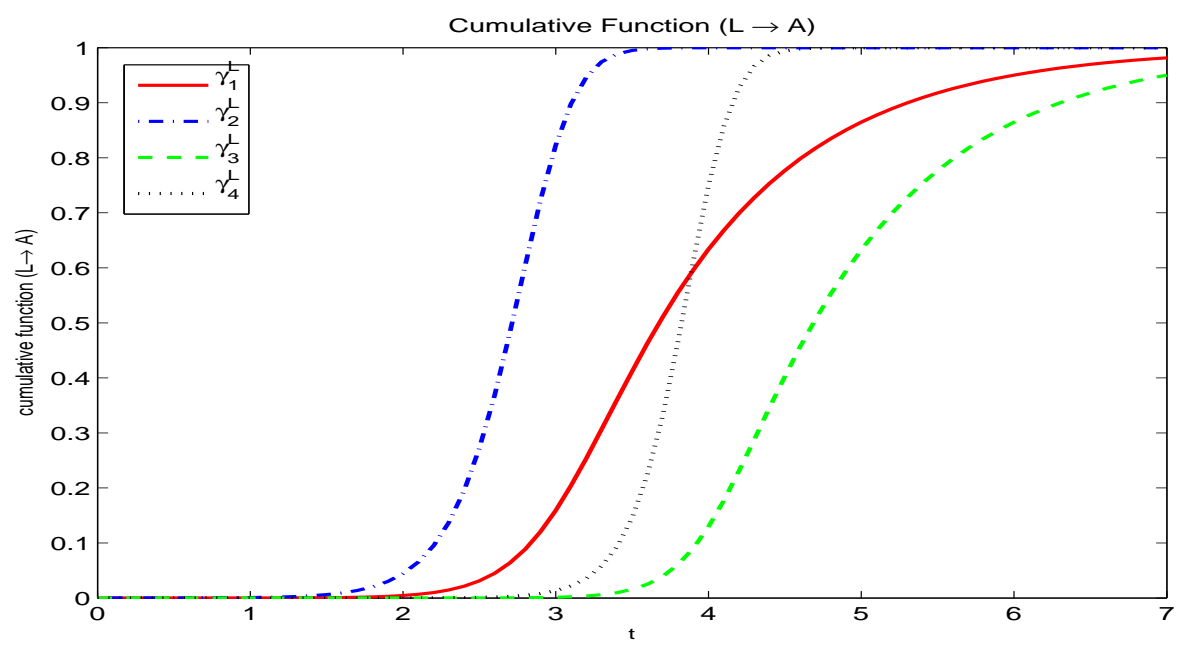

Figure 16: The cumulative latent to acute functions. 



Figure 17: Results for the latent population $L$. 

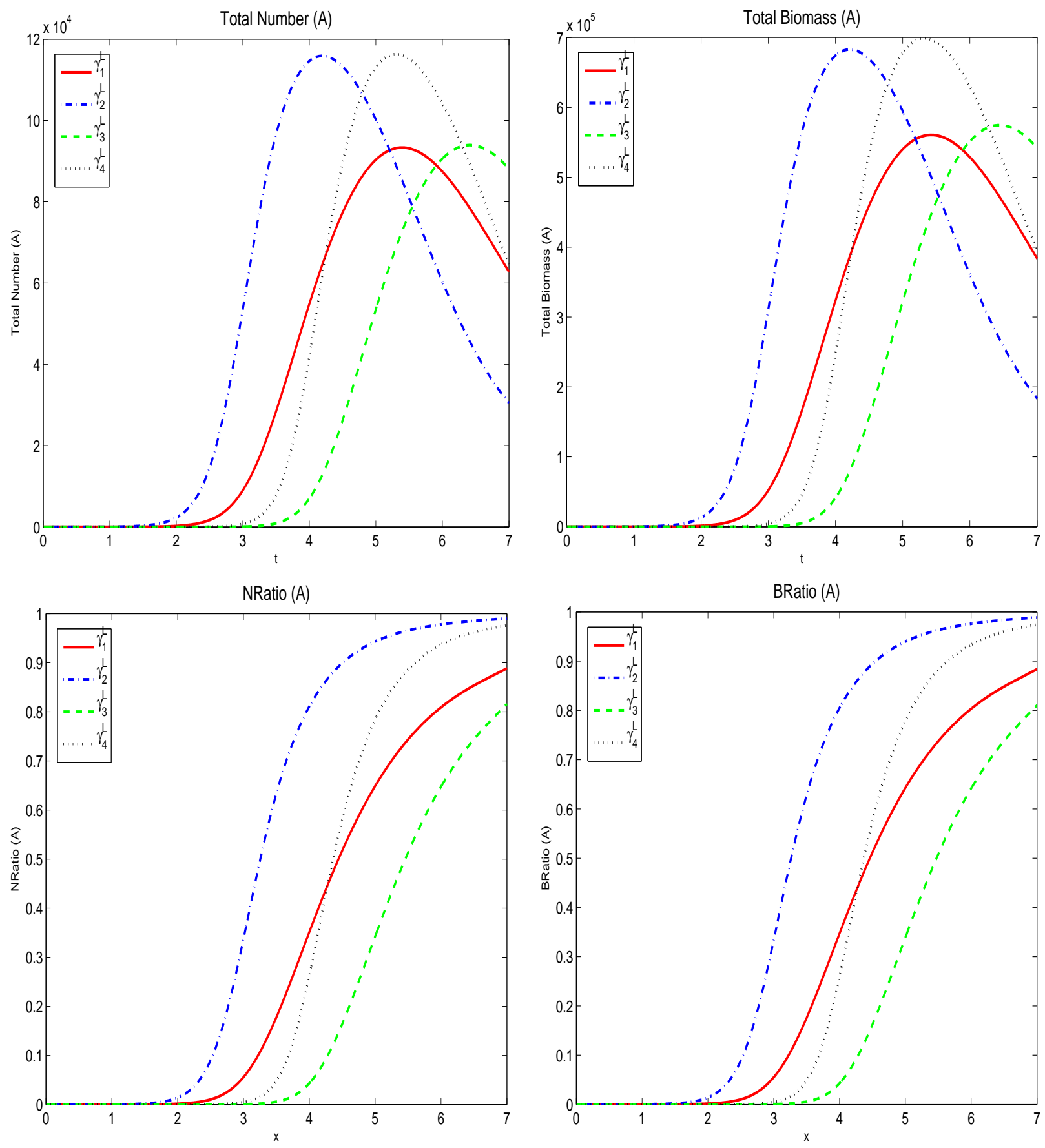

Figure 18: Results for the acute population $A$. 


\section{Conclusions and Future Work}

In this paper we have reported on our initial efforts to develop quantitative models along with numerical techniques for simulation of a coupled biomass/vaccine production system. While the overall approach is valid for general viruses in crustaceans, we focus here on Taura Syndrome Virus in controlled shrimp populations. After developing the models with detailed and explicitly stated assumptions, we demonstrate their utility in understanding possible outcomes in terms of biomass ratios for several latent to acute rate functions $\gamma^{L}$. Our numerical simulations in Section 5 indicate that the distribution of the infected population in the latent and acute stages is very sensitive to the shape and magnitude of the function $\gamma^{L}$. Hence $\gamma^{L}$ plays an important role in the construction and operation of a production system such as the raceways mentioned in our introduction. One should also understand sensitivity of the model with respect to other functions such as the mortality rate $m^{A}$ since understanding these will assist in determining the optimal harvest time of infected shrimp in order to obtain maximal production of vaccine/antibody. Once these parameters are known, one can use control theoretic methods to design efficient production systems. We note that, in general, parameters such as $\gamma^{L}$ and $m^{A}$ will be species dependent as well as virus specific. A more formal mathematical sensitivity analysis methodology for the models we have investigated can be developed using ideas from $[2,5]$ in a Prohorov metric framework.

To estimate these critical parameters, we will need to develop an inverse problem methodology which will also help in ascertaining feasibility and the practical implications of various operating conditions in the production system. We will also need data obtained on a small scale from a population of shrimp infected with a specific virus so that an inverse problem can be performed to estimate $\gamma^{L}, m^{A}$, and other important parameters of the production system. We can then use the estimated parameters to carry out forward simulations using the coupled biomass and vaccine production model developed in this paper to determine efficient ways to operate the raceway.

There are other parameters in our coupled biomass and vaccine production model that also have a significant impact on the outcome of the production system. In this paper we have fixed the growth rates and the mortality rates in the biomass model as well as in all three compartments in our vaccine model. This was done on the assumption that the shrimp populations grow and are infected in a controlled environment. Indeed parameters such as nutrient levels, temperature and the density of the population, which can significantly affect the growth and mortality rates, can all be controlled to some degree. In order to ascertain how these quantities affect the dynamics of the production system it will be necessary to obtain data for mortality in shrimp affected by a particular virus as well as growth and mortality of normal shrimp in controlled environments. The infection rate $\lambda$ may also be controlled in the production system since one can introduce chopped infected biomass into the raceway to allow all the shrimp to become infected in a certain number of days. In this paper we fixed the infection rate $\lambda$ to be a constant and it thus is only determined by a factor of ingestion. This was done on the assumption that all the shrimp have an equal chance to become infected and all other modes of transmission of virus are negligible compared to infection via ingestion. In order to determine the optimal amount of chopped infected biomass required to infect the entire shrimp population in a certain period of time, we can use inverse problem techniques along with data obtained from experiments.

In the section on data fitting we have shown that data for normal growth of shrimp 
obtained from the Waddell Mariculture center could be fairly approximated with linear functions in the range 1-20 gm. As mentioned in Section 2, exponential growth of early postlarvae has been observed by authors in [12]. Thus, it may be that production of vaccine could be significantly higher from a given biomass of postlarvae vs. the same biomass of juvenile or adult shrimp. Hence an important task involves developing a second separate model for nursery phase of shrimp growth from zero to one gram to explore use of very small animals as production system for vaccine or protein. This could be compared to the existing model. We would have to know the differences in dynamics of infection of small shrimp vs. juveniles and possible differences in viral load per gram body mass as shrimp become larger. This would be an interesting application of the models for comparison, assuming we can answer some of the basic questions with a reporter gene or endogenous viral protein in bioassay studies. Clearly, one would need additional data in order to investigate the importance of exponential growth in our model.

Our model and associated computational methodology are sufficiently general to permit future investigation of both underlying crustacean growth/death hypotheses and marine raceway operating conditions as well as characteristics of viral progression in specific species, all of which are important to the marine aquaculture industry. For the study of viral epidemiology of marine species, the calculation of the basic reproduction ratio, usually denoted by $R_{0}$ in the literature [20], is important to determine whether the viral disease persists or dies out. Our interest in this paper is vaccine production, and calculation of $R_{0}$ is not important in this context. Computation of $R_{0}$ could in principle, of course, be done for models such as ours (e.g., see [16]), but we do not pursue that here.

Investigation of commercial aspects of the overall countermeasure production system can also be pursued using this class of models with theoretic control methods.

\section{Acknowledgements}

This research was supported in part by the Defense Advanced Research Projects Agency (DARPA) under grant ARO W911NF-05-1-0230, in part by the Joint DMS/NIGMS Initiative to Support Research in the Area of Mathematical Biology under grant 1R01GM67299-01, and in part by the U.S. Air Force Office of Scientific Research under grant AFOSR-FA9550-04-1-0220. The authors are also grateful to Dr. Anna Tsao of AlgoTek, Inc. for her initial efforts in promoting the joint efforts of the groups involved in this research. The authors note that the production system described in this paper has a patent applied for and published in PCT as International Publication WO 03/024482 A1 and in the USPTO as US Patent Application 20040177392.

\section{References}

[1] M. Adimy and L. Pujo-Menjouet, A mathematical model describing cellular division with a proliferating phase duration depending on the maturity of cells, Elect. J. Diff. Equations, 2003 (2003), 1-14. 
[2] H.T. Banks and D.M. Bortz, Inverse problems for a class of measure dependent dynamical systems. Journal of Inverse and Ill-posed Problems, 13 (2005), 103121.

[3] H.T. Banks, F. Kappel and C. Wang, Weak solutions and differentiability for sizestructured population models, International Series of Numerical Mathematics, 100 (1991), 35-50.

[4] H.T. Banks and F. Kappel, Transformation semigroups and $L^{1}$-approximation for size- structured population models, Semigroup Forum, 38 (1989), 141-155.

[5] H.T. Banks and H.K. Nguyen, Sensitivity of dynamical systems to Banach space parameters. CRSC Technical Report, CRSC-TR05-13, NC State University, Raleigh, 2005; Journal of Mathematical Analysis and Applications, in press.

[6] H.T. Banks and I.G. Rosen, Spline approximations for linear nonautonomous delay systems, J. Math. Analysis and Applications, 96 (1983), 226-268.

[7] C.L. Browdy and S.M. Moss, Shrimp culture in urban, super-intensive closed systems, Chapter 11 in Urban Aquaculture, B. Costa-Pierce, A. Desbonnet, P. Edwards and D. Baker, eds., CABI Publishing, Cambridge, MA, 2005, 173-185.

[8] D.P. Bureau, P.A. Azevedo, M. Tapia-Salazar and G. Cuzon, Pattern and cost of growth and nutrient deposition in fish and shrimp: Potential implications and applications, in Potential Implications and Applications. Avances en Nutrición Acuicola V: Memorías del Quinto Simposium Internacional de Nutrición Acucola, 19-22 Nov., 2000, Mérida, Yucatan, Mexico.

[9] S.X. Cadrin, J.A. Boutillier and J.F. Idoine, A hierarchical approach to determining reference points for Pandalid Shrimp, Can. J. Fish. Aquat. Sci, 61 (2004), $1373-1391$.

[10] A. Cartaxana, Growth of the prawn Palaemon Longirostris (Decapoda, Palaemonidae) in Mira River and Estuary, SW Portugal. J. Crustacean Biol., 23 (2003), $251-257$.

[11] R. Carvajal and A. Nebot, Growth model for White Shrimp in semi-intensive farming using inductive reasoning methodology, Computers and Electronics in Agriculture, 19 (1998), 187-210.

[12] F.L. Castille, T.M. Samocha, A.L. Lawrence, H. He, P. Frelier and F. Jaenike, Variability in growth and survival of early postlarval shrimp (Penaeus vannamei Boone 1931) Aquaculture, 113 (1993), 65-81.

[13] A.K. Dhar, J.A. Cowley, K.W. Hasson and P.J. Walker, Genomic organization, biology, and diagnosis of Taura Syndrome Virus and Yellohead Virus of Penaeid Shrimp, Advances in Virus Research, 63 (2004), 353-420.

[14] K.W. Hasson, D.V Lightner, L.L. Mohney, R.M. Redman, B.T. Poulos and B.M. White, Taura Syndrome Virus (TSV) lesion development and the disease cycle in the Pacific white shrimp Penaeus vannamei, Diseases of Aquatic Organisms, 36 (1999), 81-93.

[15] F. Hoppensteadt, An age dependent epidemic model, J. Franklin Inst., 297 (1974), $325-333$.

[16] H. Inaba and H. Sekine, A mathematical model for Chagas disease with infectionage-dependent infectivity, Mathematical Biosciences, 190 (2004), 39-69. 
[17] C.J. Jackson and Y-G. Wang, Modelling growth rate of Penaeus Monodon Fabricius in intensively managed ponds: Effects of temperature, pond age and stocking density, Aquaculture Research, 29 (1998), 27-36.

[18] Mark Kot, Elements of Mathematical Ecology, Cambridge University Press, Cambridge, 2001.

[19] S.G. Krein, Linear Differential Equations in Banach Space, Translations Math. Monographs, Vol. 29, American Math Society, Providence, RI, 1971.

[20] J.M. Lotz, A.M. Flowers and V. Breland, A model of Taura Syndrome Virus (TSV) epidemics in Litopenaeus Vannamei, J. Inver. Path., 83 (2003), 168-176.

[21] J.A.J. Metz and E.O. Diekmann, The Dynamics of Physiologically Structured Populations, Lecture Notes in Biomathematics, Vol. 68, Springer, Heidelberg, 1986.

[22] E. Sinestrari and G. Webb, Nonlinear hyperbolic systems with nonlocal boundary conditions, J. Math. Analysis and Applic., 121 (1987), 449-464.

[23] J.W. Sinko and W. Streifer, A new model for age-size structure of a population, Ecology, 48 (1967), 910-918.

[24] D.L. Taylor and S. Collie, A temperature and size-dependent model of sand shrimp (Crangon septemspinosa) predation on juvenile winter flounder (Pseudopleuronectes americanus) Can. J. Fish. Aquat. Sci, 60 (2003), 1133-1148. 